\title{
A modular and flexible ESC-based mouse model of pancreatic cancer
}

\author{
Michael Saborowski, ${ }^{1}$ Anna Saborowski, ${ }^{1}$ John P. Morris IV, ${ }^{1}$ Benedikt Bosbach, ${ }^{1}$ Lukas E. Dow, \\ Jerry Pelletier, ${ }^{2}$ David S. Klimstra, ${ }^{1}$ and Scott W. Lowe ${ }^{1,3,4}$ \\ ${ }^{1}$ Memorial Sloan-Kettering Cancer Center, New York, New York 10065, USA; ${ }^{2}$ McGill University, Montreal, Quebec H3A OG4, \\ Canada; ${ }^{3}$ Howard Hughes Medical Institute, New York, New York 10032, USA
}

\begin{abstract}
Genetically engineered mouse models (GEMMs) have greatly expanded our knowledge of pancreatic ductal adenocarcinoma (PDAC) and serve as a critical tool to identify and evaluate new treatment strategies. However, the cost and time required to generate conventional pancreatic cancer GEMMs limits their use for investigating novel genetic interactions in tumor development and maintenance. To address this problem, we developed flexible embryonic stem cell (ESC)-based GEMMs that facilitate the rapid generation of genetically defined multiallelic chimeric mice without further strain intercrossing. The ESCs harbor a latent Kras mutant (a nearly ubiquitous feature of pancreatic cancer), a homing cassette, and other genetic elements needed for rapid insertion and conditional expression of tetracycline-controlled transgenes, including fluorescence-coupled shRNAs capable of efficiently silencing gene function by RNAi. This system produces a disease that recapitulates the progression of pancreatic cancer in human patients and enables the study and visualization of the impact of gene perturbation at any stage of pancreas cancer progression. We describe the use of this approach to dissect temporal roles for the tumor suppressor Pten and the oncogene $c-M y c$ in pancreatic cancer development and maintenance.
\end{abstract}

[Keywords: pancreatic cancer; mouse model; kras; pancreas; RMCE; embryonic stem cell]

Supplemental material is available for this article.

Received October 10, 2013; revised version accepted December 4, 2013.

Cancer is a multistep disease involving complex mutational interactions that underlie the heterogeneous behavior of tumors in patients (Hanahan and Weinberg 2011). Indeed, recent genomic efforts have enabled "cataloging" of the many genes and gene combinations that contribute to various cancers, and these data argue that virtually every human tumor is unique (Vogelstein et al. 2013). While these analyses have also pointed toward more rational approaches to treating human cancer, genomic studies alone are insufficient to determine whether and how somatic mutations observed in human cancers act as "drivers" of the disease or create vulnerabilities that can be targeted directly or indirectly for therapeutic gain. In addition, advances in cancer biology illustrate the importance of the tumor microenvironment in tumor initiation and progression as well as tumor response to various anti-cancer therapies (Barcellos-Hoff et al. 2013).

${ }^{4}$ Corresponding author E-mail lowes@mskcc.org

Article is online at http://www.genesdev.org/cgi/doi/10.1101/gad.232082.113. Freely available online through the Genes \& Development Open Access option.
Thus, while our understanding of the cancer genome provides a strong rationale for personalized cancer therapy, in vivo models are needed to fully understand how cancer drivers or dependencies impact tumor initiation and maintenance.

Genetically engineered mouse models (GEMMs) have proven to be a powerful tool to study the impact of gene function on tumorigenesis (for review, see Frese and Tuveson 2007; Dow and Lowe 2012). Over the last three decades, genome engineering and stem cell technologies have enabled the production of mouse strains in which individual genes can be ectopically expressed in a tissuespecific manner or altered at their endogenous locus through homologous recombination. With the advent of Cre-lox technology, conditional knockout or knockin alleles can precisely model the mutational events associated with human disease. Moreover, conditional 
gene expression systems based on the tetracycline (tet)based response system or estrogen-receptor fusions allow analysis of the temporal requirement for gene function, and incorporation of fluorescent reporters enables lineage tracing and isolation of specific cell types from complex tissues. By combining these approaches, it has been possible to produce genetically and histopathologically accurate mouse models of various human cancers that in turn have been used extensively to study all aspects of cancer biology and therapy response.

Pancreatic ductal adenocarcinoma (PDAC) is a cancer type in which GEMMs have proven particularly informative. PDAC is incurable, with patients often presenting with a metastatic disease that is resistant to all current therapies (Hidalgo 2010). Genomic studies reveal that activating mutations in KRAS are almost an ubiquitous event, although inactivating mutations in P53 and SMAD4 and at the INK4A/ARF locus are also common. Histopathologically, the disease progresses through a stepwise process of precursor pancreatic intraepithelial neoplasia (PanIN) lesions to PDAC, characterized by a massive desmoplastic stromal reaction, hypovascularity, and extensive immune cell infiltration. GEMMs in which a latent "lox-stop-lox" Kras ${ }^{\text {G12D }}$ allele is activated using a pancreas-specific Cre recombinase recapitulate all of these histological characteristics (Hingorani et al. 2003), and models that incorporate additional relevant lesions such as p53 mutations show accelerated disease onset and metastasis (Aguirre et al. 2003; Hingorani et al. 2005; Bardeesy et al. 2006a,b). These models have helped validate new genes as drivers of PDAC development or metastasis, shown the importance of the PDAC tumor microenvironment on disease progression, and been used as preclinical models to study therapy response /Olive et al. 2009; Ardito et al. 2012; Provenzano et al. 2012; Eser et al. 2013; Jacobetz et al. 2013).

Despite the power of genetically engineered mice, they are slow and expensive to produce and analyze, and methods to interrogate gene function in established cancers are cumbersome and inefficient. Consequently, it is unrealistic to use GEMMs to develop models that approximate the extent of heterogeneity that exists in human cancers or as an essential component of the target validation process. For example, in the case of the KPC model (LSL-Kras ${ }^{G 12 D}$, LSL-p53 ${ }^{R 172 H}$, and Pdx1-Cre) (Hingorani et al. 2005), the analysis of an additional gene on PDAC development may involve producing multiallelic mice harboring five engineered alleles and time-consuming intercrossing. Furthermore, the introduction of gene deletion upon Cre-activation during embryogenesis can result in developmental defects that cause early lethality or impact pancreas development, confounding assessment of their requirement in PDAC (Nakhai et al. 2008; Bonal et al. 2009; Mazur et al. 2010). Finally, such approaches cannot assess the requirement for gene function in tumor maintenance, which is the gold standard for validating new cancer drug targets. As a consequence, GEMMs of PDAC are underused, and often the contribution of only one or two genes to the disease can be assessed in any given laboratory. Nonetheless, the need to better implement
GEMMs is evidenced by observations that human cells and even xenografts show distinct behavior compared with pancreas tumors arising in their natural site in an immunocompetent environment (Olive et al. 2009).

Our laboratory has taken several steps to accelerate the pace at which gene function can be interrogated in mice. In one line of research, we optimized the application of RNAi as a rapid alternative to gene deletion by homologous recombination. Using shRNAs, we showed that gene suppression by RNAi can mimic loss of gene function in mice (Hemann et al. 2003; Dickins et al. 2005, 2007) and, more recently, adopted an efficient transgenesis approach that implements a recombinase-mediated cassette exchange strategy (RCME) to introduce tet-responsive shRNA expression cassettes into embryonic stem cells (ESCs) at a defined genomic locus (Premsrirut et al. 2011; Dow et al. 2012). By coupling the shRNA to a fluorescent reporter, it is possible to track and isolate shRNA-expressing cells in tissues and monitor target gene knockdown (Premsrirut et al. 2011; Zuber et al. 2011). We used these systems to study the role of tumor suppressor gene loss in tumor maintenance and the role of an essential gene and define functional requirements for gene expression at different stages of development (McJunkin et al. 2011; Premsrirut et al. 2011).

In a complementary line of investigation, we and others are developing ESC models harboring multiple alleles needed to model a disease phenotype without further intercrossing (Heyer et al. 2010; Zhou et al. 2010; Huijbers et al. 2011; Premsrirut et al. 2011). Our approach is based on the derivation of ESC lines that harbor conditional disease-associated alleles, which, in combination, generate functional mouse cancer models. These "GEMM-ESCs" can be manipulated in vitro (to introduce additional genetic complexity) and used to produce cohorts of experimental mice by blastocyst injection or tetraploid complementation. Thus, the GEMM-ESC approach in combination with inducible shRNA-mediated gene silencing enables the production of experimental animals in $<2$ mo from the time of the initial ESC targeting event (Supplemental Fig. S1). This accelerates functional analysis and potentially reduces costs by eliminating the additional breeding steps required for establishing germline transmission and obtaining study cohorts with conventional transgenic techniques. Moreover, as additional genetic alterations can be introduced in cell culture, many different genes can be assessed in parallel without the need for large, expensive breeding colonies. Here, we produce GEMM-ESC models that enable the rapid analysis of a particular gene or gene combination on PDAC development, progression, and maintenance.

\section{Results}

\section{Generation of pancreatic GEMM-ESCS}

We reasoned that GEMM-ESC models that harbor all of the elements for pancreas-specific activation of an established PDAC driver and inducible expression of shRNAs would provide a powerful platform for rapidly characterizing 
additional PDAC drivers and validating therapeutic targets. To do this, we derived two mouse ESC lines, each containing four defined alleles: (1) LSL-Kras ${ }^{G 12 D}$ (Jackson et al. 2001), permitting Cre-mediated expression of oncogenic Kras ${ }^{\mathrm{G} 12 \mathrm{D}}$; (2) Pdx1-Cre or p48-Cre, to enable pancreas-specific activation of Cre-dependent alleles (Kawaguchi et al. 2002; Hingorani et al. 2003); (3) col1a1 RMCE-based "homing cassette" (hereafter referred to as $C H C$ ), which enables high-efficiency targeting with tetregulatable shRNAs or cDNAs (Beard et al. 2006; Premsrirut et al. 2011; Dow et al. 2012); and (4) a new Cre-dependent tet transactivator, CAGs-LSL-rtTA $-I R E S$ mKate2 (CAGs-LSL-RIK), allowing doxycycline (dox)dependent induction of TRE-controlled elements and expression of fluorescent mKate2 in cells that have undergone Cre recombination (Fig. 1A). Pdx1-Cre and p48-Cre display distinct Cre expression patterns such that Pdx1-Cre shows dispersed expression in the pancreas (Hingorani et al. 2003), whereas p48-Cre is expressed in the majority of exocrine cells (Heiser et al. 2008). We therefore envisioned that $P d \times 1$-Cre would facilitate mosaic activation of $\mathrm{Kras}^{G 12 D}$ and shRNA expression, allowing tumor development in the context of normal pancreatic tissue, while $p 48$-Cre would enable tissue-wide $\mathrm{Kras}^{\mathrm{G} 12 \mathrm{D}}$ induction, facilitating quantitative analysis of early acinar-to-ductal metaplasia (ADM) and early grade PanIN formation.

Mouse strains maintained primarily on the C57BL/6 background harboring the alleles described above were intercrossed to produce multiallelic animals, after which ESCs were rederived in the presence of a MEK and a GSK3 $\beta$ inhibitor (PD0325901 and CHIR99021) (Gertsenstein et al. 2010). Clones with a good ESC morphology were expanded and established into individual lines. Four male ESC lines harboring all four desired alleles were used to produce chimeric mice by injection into blastocysts derived from C57BL/6 albino mice (Fig. 1A). Each clone gave rise to chimeric recipient mice, although the number of offspring arising and the percent chimerism varied between clones (for examples, see Supplemental Fig. 2B-E). Fluorescence analysis of the isolated organs revealed mKate2 expression in the pancreas (Fig. 1B), indicating that the cre transgene successfully recombined the CAGs-LSL-rtTA ${ }_{3}$-IRES-mKate2 allele, leading to its transcriptional activation. Similarly, all mice showed some degree of ADM and PanINs in the pancreas, implying that the Kras ${ }^{G 12 D}$ allele was activated (for example, see Fig. 2A).

For further analysis, we chose an ESC line that produced high numbers of chimeric offspring that developed pancreatic lesions closely resembling those found in germline transgenic Pdx1-Cre or p48-Cre; LSL-Kras ${ }^{G 12 D}$ mice. These ESC lines were karyotyped and tested for strain contribution using a 384 single-nucleotide polymorphism (SNP) panel. Each ESC clone showed a diploid profile, and, as expected, the GEMM-ESCs predominantly harbored C57BL/6 alleles and a small percentage of 129 alleles $(\sim 94 \%$ and $82 \%$ C57BL/6-derived alleles and $6 \%$ and $18 \%$ 129-derived alleles for Pdx1-Cre- and p48-Crecontaining ESCs, respectively). These results were confirmed by high-density array comparative genomic hybridization (CGH) analyses against C57BL/6J reference DNA, which detected only a few, predominantly focal, copy number variations (CNVs) (Supplemental Table 1). The Pdx1 GEMM-ESCs successfully generate, on average, 16 (plus or minus three) chimeric mice when injected by regular blastocyst injection (50-60 transferred blastocysts), whereas the p48 GEMM-ESCs produce, on average,
A

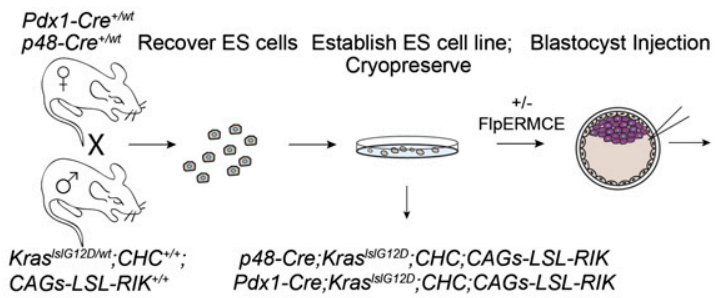

B

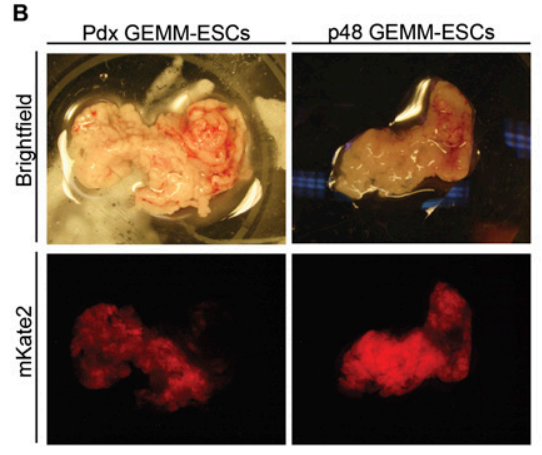

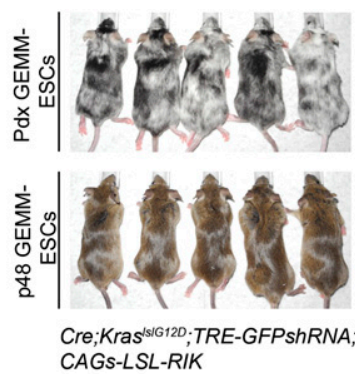

C

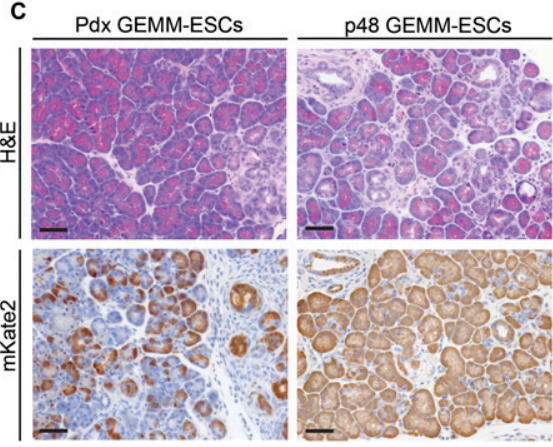

Figure 1. Development of ESC-based RMCEcompetent mouse models of pancreatic cancer. (A) Technical outline: ESC lines were established from multiallelic transgenic mice and targeted by Flp/Frt-mediated recombination. Chimeric mice were generated from the untargeted ESC line or targeted ESC clones by injection into albino $\mathrm{B} 6$ blastocysts. The p48 GEMM-ESC-derived mice are characterized by high ESC contribution as assessed by coat color (agouti). For comparison, mice shown in this figure were all scored $>95 \%$ coat color chimerism. From left to right, the Pdx GEMM-ESC animals with black coat color were scored $40 \%, 30 \%$, $15 \%, 60 \%$, and $10 \%$. (B) Bright-field and fluorescence images of pancreata of mice derived from both GEMM-ESCs expressing mKate2. $(C)$ H\&E and mKate immunohistochemistry of pancreata from mice generated by the Pdx1 GEMM-ESCs and the p48 GEMM-ESCs. mKate2 expression was detected in the vast majority of exocrine cells in the p48 GEMM-ESC mice and shows a mosaic expression pattern in the $\mathrm{Pdx} 1$ GEMM-ESC animals. Bars, $50 \mu \mathrm{m}$. 

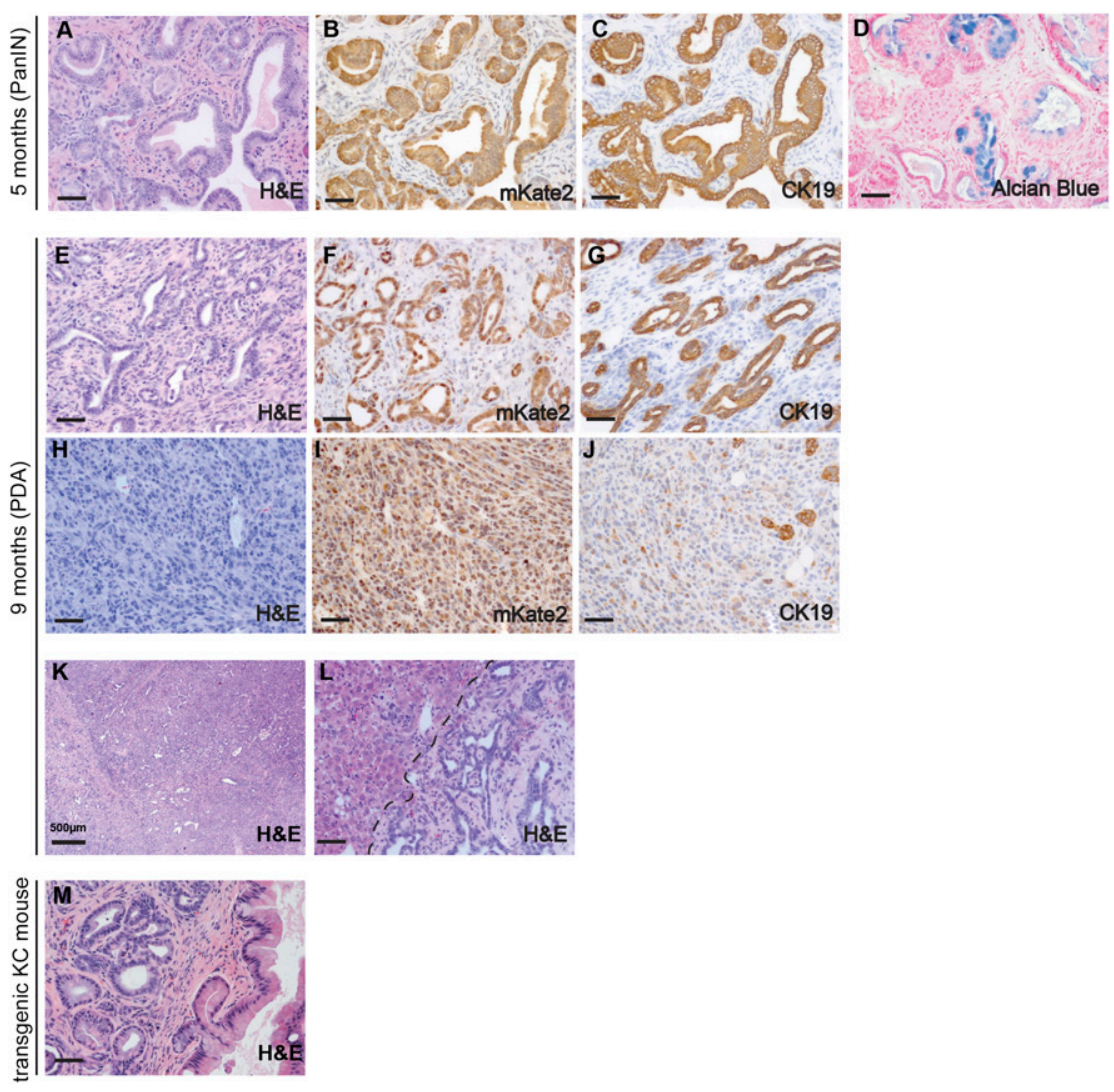

22 (plus or minus two) mice. Both ESC lines were capable of substantial contributions to the animal, with coat color contributions $>90 \%$ occurring in $33 \%$ and $71 \%$ of the Pdx1 GEMM-ESC-derived and p48 GEMM-ESC-derived mice, respectively (Supplemental Fig. S2B,C).

We examined chimeric mice at different times after birth to further assess the extent to which the GEMM-ESC model recapitulates the germline setting. For the Pdx1-Cre model, Cre activity-as visualized by mKate2 fluorescence-was detected in a subset of pancreatic cells even in mice with high GEMM-ESC contribution, presumably owing to the previously reported mosaic expression of Pdx1-Cre (Hingorani et al. 2003). In contrast, high-contribution p48 GEMM-ESC animals showed Cre activity in the majority of the exocrine pancreas (Fig. 1C). Comparable with conventional transgenic Pdx1-Cre or p48-Cre mice carrying LSL-Kras ${ }^{G 12 D}$, animals derived from our GEMM-ESCs developed ADM/PanIN lesions accompanied by the expansion of surrounding stromal cells (Fig. 2A). The majority of PanIN lesions expressed mKate2 and were positive for CK19, confirming their ductal phenotype (Fig. 2B,C). Importantly, they also produced mucin, as judged by Alcian blue staining, a histological hallmark of PanIN lesions (Fig. 2D).

We observed two pancreatic cancers in our GEMM-ESC mice at $7 \mathrm{mo}(\mathrm{p} 48)$ and $9 \mathrm{mo}(\mathrm{Pdx} 1)$ of age. Consistent with previous reports (Hingorani et al. 2003), the tumors harbored both CK19-positive well-differentiated glandular regions (Fig. 2E-G) and undifferentiated areas (Fig. 2H-J).
Figure 2. Chimeric mice generated with the PDAC GEMM-ESCs display Kras ${ }^{\mathrm{G} 12 \mathrm{D}}$ induced neoplasia. $(A-D)$ H\&E shows PanIN lesions in chimeric mice generated by the Pdx1 GEMM-ESCs. PanINs are mKate2positive, as opposed to the abundant stroma, and stain-positive for mucins (Alcian blue). Cytokeratin 19 confirmed ductal differentiation. (E-L) PDAC that developed in a 9mo-old Pdx1 GEMM-ESC mouse. $(E-G)$ Well-differentiated area from $K$ showing mKate2-expressing invasive glandular lesions. Ductal differentiation was confirmed by CK19 immunohistochemistry in $G$. $(H-J)$ Poorly differentiated area from $K$ in which cells are mKate2-expressing but mostly stainnegative for cytokeratin 19. $(L)$ Liver metastasis found in the same animal and displaying well-differentiated malignant lesions and abundant stromal desmoplasia. (M) PanINs from a mouse with pancreatic Kras ${ }^{\mathrm{G} 12 \mathrm{D}}$ expression, generated by traditional breeding for comparison. Bars, $50 \mu \mathrm{m}$ if not otherwise specified.
The Pdxl GEMM-ESC carcinoma was associated with a hepatic metastatic focus of well-differentiated adenocarcinoma (Fig. 2L). For unknown reasons, a subset of mice $(15 \%-20 \%$ and $<10 \%$ for Pdx1-Cre- and p48-Crecontaining GEMM-ESC animals, respectively) developed nonpancreatic illnesses (see the Materials and Methods) and were excluded from further analysis. Regardless, both GEMM-ESCs provide a platform to rapidly assess the function of genes in mediating the early stages of PDAC.

\section{Validating cancer drivers in pancreatic cancer initiation and maintenance}

To determine whether these GEMM-ESC models could be used for validating cancer drivers and assessing their role in tumor maintenance, we chose to investigate whether suppression of the Pten tumor suppressor cooperates with Kras ${ }^{\mathrm{G} 12 \mathrm{D}}$ during pancreatic cancer formation and, if so, whether ongoing Pten suppression was required to maintain disease. PTEN is a lipid phosphatase that acts to dephosphorylate phosphatidylinositol $(3,4,5)$ triphosphate (PIP3) and thus serves as a negative regulator of the phosphatidylinositide 3-kinase (PI3K) pathway (Cantley 2002). Heterozygous deletions encompassing PTEN (Ying et al. 2011) as well as activating mutations in the PI3K pathway (Schonleben et al. 2006; Jaiswal et al. 2009) occur in PDAC, and, accordingly, inactivation of Pten accelerates the development of PDAC in combination 
with $\operatorname{Kras}^{G 12 D}$ (Hill et al. 2010; Ying et al. 2011). As components of the PI3K pathway are the target of drugs in clinical trials, experiments assessing the impact of genetic PI3K pathway inhibition in established cancers should anticipate the effectiveness of pharmacologic on-target pathway inhibition.

We targeted the Pdx1 GEMM-ESCs with a potent shRNA directed against Pten (Supplemental Fig. S3A; see Fellmann et al. 2011 for shRNA validation). Correct, single-copy integration of the TRE-GFP-shRNA cassette to the CHC locus was confirmed by allele-specific PCR and then GFP copy number assays to eliminate clones with additional random integrations. In parallel, ESC clones were tested for proper GFP induction upon $\mathrm{rtTA}_{3}$ activation by adenoviral Cre recombinase and subsequent dox treatment in vitro (see the Materials and Methods). We did not detect general activation of the latent $\mathrm{rtTA}_{3}$ by Pdx1-Cre (or p48-Cre) in dox-only-treated targeted ESC clones. We then used the targeted cells (referred to as shPten ESCs) to produce chimeric mice, which were treated with dox (in chow) from postnatal day 5 to induce the Pten shRNA. Around $45 \%$ of chimeras developed palpable tumors within 5 mo of dox addition (see below). Such effects were not observed in dox-treated chimeric mice derived from the same ESCs expressing a potent control shRNA targeting renilla luciferase (shRen), indicating that neither dox treatment nor shRNA expression per se were responsible for the observed morbidity (Supplemental Fig. S4A).

Abdominal palpation, ultrasound, and various histological analyses of dox-treated mice derived with shPten ESCs revealed the presence of large pancreatic masses that were highly fibrotic. These lesions consisted of ADM, high-grade PanINs, and locally invasive carcinoma similar to tumors found in transgenic Pdx1-Cre; LSLKras $^{G 12 D}$; Pten ${ }^{\text {flox/flox }}$ mice (Fig. 3A, top left; Ying et al. 2011). Pten protein suppression was specifically observed in the epithelial compartment, which also coexpressed GFP and mKate2 reporters coupled to the shRNA and $\mathrm{rtTA}_{3}$ transcripts, respectively (Fig. 3A, top panel). Thus, the Pdx1 GEMM-ESC model can be used to specifically drive shRNA expression in the pancreas to potently repress gene expression and recapitulate the effects of gene deletion.

We next examined whether dox withdrawal could silence the shRNA, restore Pten expression, and produce an anti-cancer effect. Chimeric mice derived from shPten ESCs were treated with dox and, upon tumor formation
A

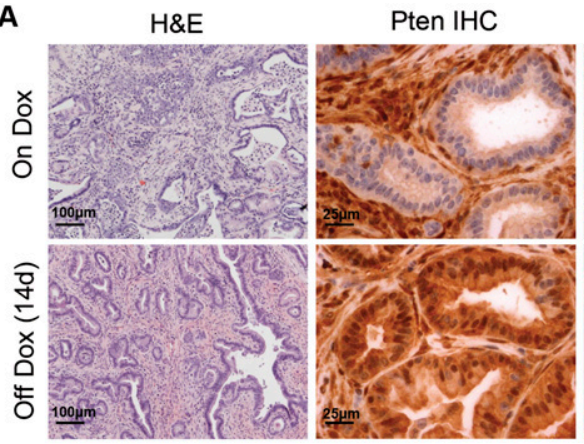

B

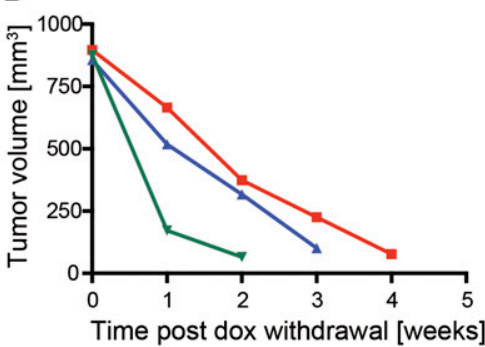

mKate2 (IF)

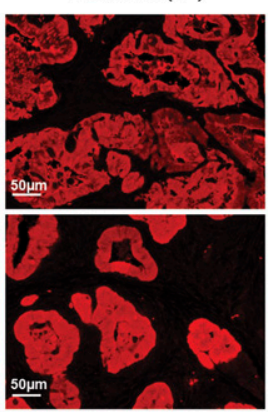

\section{C}

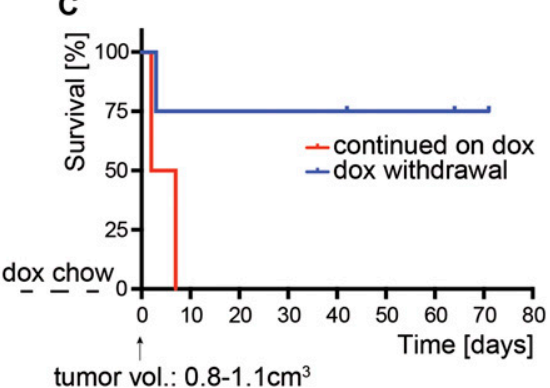

GFP (IF)

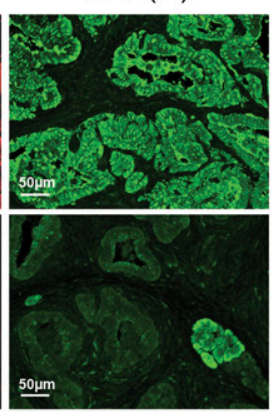

D
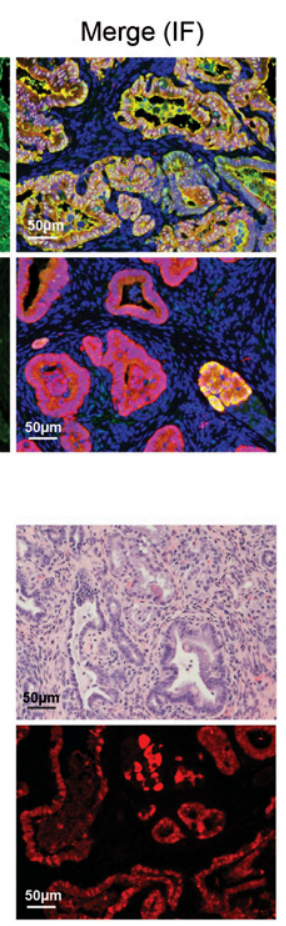

Figure 3. Pten is required for tumor maintenance, and its restoration leads to rapid tumor regression and a significant survival benefit. (A) On dox versus off-dox (14 d) comparison of pancreatic tumors from two shPten mice. H\&E shows area of focal invasive pancreatic cancer in the top panel. Pten immunohistochemistry confirmed Pten depletion in the on-dox mouse within the Pdxl-Cre recombined neoplastic lesions and restoration of Pten expression in the off-dox mouse. Immunofluorescence analysis showed persistent mKate2 expression and dox-dependent GFP expression. The only remaining pancreatic cells with strong EGFP expression in the off-dox tumor were morphologically normal acinar cells, likely due to a low turnover rate and retention of EGFP protein. $(B)$ Rapid tumor regression upon restoration of Pten expression in mice taken off dox food as assessed by three-dimensional ultrasound imaging (each line represents one individual mouse). $(C)$ KM survival of shPten mice randomized into on-dox $(n=4)$ and off-dox $(n=4)$ treatment arms after detection of an 800- to $1100-\mathrm{mm}^{3}$ pancreatic tumor. (D) Four weeks to 6 wk after dox deprivation, pancreata still harbor mKate2positive neoplastic lesions. 
(as assessed by palpation and/or ultrasound), withdrawn from dox and monitored over time. By 2 wk after dox withdrawal, Pten protein levels were fully restored in the epithelial compartment, which corresponded to silencing of the shRNA-linked GFP reporter (Fig. 3A, bottom panel). The only remaining pancreatic cells with strong GFP expression were morphologically normal acinar cells, likely due to low turnover rate and retention of GFP protein. Notably, mKate2 fluorescence was independent of dox treatment, highlighting the utility of the mKate 2 reporter to identify Cre-recombined cells that had expressed the shRNA even after dox withdrawal.

The effects of Pten restoration on tumor progression and survival were dramatic. Whereas tumor-bearing mice maintained on dox exhibited persistent signs of exocrine insufficiency such as steatorrhea and bloating of the caecum and became moribund in $<10 \mathrm{~d}$, withdrawal of dox produced dramatic tumor shrinkage (Fig. 3B; Supplemental Fig. S4B) and increased survival (Fig. 3C). Tumor volume was followed weekly until masses shrank below $150 \mathrm{~mm}^{3}$ and remained undetectable by palpation for the duration of the study. Despite the decrease in tumor size, mKate $2^{+}$neoplastic glands and $\mathrm{mKate} 2^{-}$desmoplastic stroma could still be found within the pancreas $4-6 \mathrm{wk}$ after removal of dox (Fig. 3D), implying that the constitutive activation of Kras can sustain a low level of neoplastic growth. These studies demonstrate that Pten reactivation triggers marked anticancer effects and imply that drugs having impact on the PI3K pathway should have activity against PDAC in similar contexts. More generally, this example illustrates how the GEMM-ESC model can be used to validate cancer drivers and therapeutic targets.

\section{Interrogating early stages of PDAC development}

We also tested whether the GEMM-ESC models could be used to determine the requirement for gene function during different stages of PDAC progression. Typically, such studies are designed to delete the test gene during embryogenesis when the Kras mutant is activated. However, such an approach can be confounded in situations where the test gene is needed for pancreas development, as has been observed for the c-Myc oncoprotein (Nakhai et al. 2008; Bonal et al. 2009; Mazur et al. 2010). In principle, using shRNAs under the control of dox regulation, it is possible to delay $c-M y c$ inactivation until after pancreas development and more effectively assess its role in the early stages of the disease. To test this directly, we used the p48 GEMM-ESCs to generate chimeric mice harboring a control Renilla shRNA (shRen) (Zuber et al. 2011), a control Kras shRNA (shKras) (Supplemental Fig. 5; Fellmann et al. 2011), or two independent shRNAs targeting $c-M y c$ (shMyc-1 and shMyc-2) (Fig. 4B; Fellmann et al. 2011).

Mice were treated with dox for $28 \mathrm{~d}$ starting postnatal day 11 , a time point at which pancreatic lineages have already been specified (Gittes 2009). Histological examination of shRen mice revealed ADM/PanIN development accompanied by vast stromal fibrosis. In contrast, mice with pancreas-specific knockdown of Kras showed largely normal pancreatic histology with only rare and small areas of ADM/PanIN (Fig. 4A). This was also reflected by a significant increase in intact acinar tissue in the shKras mice (Fig. 4C). While c-Myc repression led to a significant increase of normal acinar cells compared with shRen mice (Fig. 4C), $c-M y c$ silencing did not prevent development of ADM, early stage PanIN lesions, or stromal expansion as potently as shKras (Fig. 4A). Consistent with the histology, shRen mice showed an increase in pancreas weight that was ameliorated by suppression of either Kras or c-Myc (Fig. 4D). Together, these data imply that endogenous levels of c-Myc play a major role in $\mathrm{Kras}^{\mathrm{G} 12 \mathrm{D}}$-induced ADM/PanIN development.

\section{Studies to investigate the genetic requirements} for Kras ${ }^{G 12 D}$-induced pancreatic cancer

We also assessed whether the PDAC GEMM-ESCs could be used to study the requirements for frank pancreatic cancer development. Only a small fraction of mice with pancreas-specific expression of Kras ${ }^{\text {G12D }}$ develop pancreatic cancer within the first year (Hingorani et al. 2003), making it difficult to study the requirement of a gene for PDAC development due to a long latency and a small number of cancer-harboring mice. The addition of further genetic alterations like the loss of the tumor suppressor gene $p 53$ can dramatically accelerate pancreatic cancer development in this model (Hingorani et al. 2005; Bardeesy et al. 2006a). We took advantage of the efficient cooperation between $\mathrm{Kras}^{\mathrm{G} 12 \mathrm{D}}$ and p53 loss to generate a system that enables the rapid experimental testing of genetic requirements for in vivo pancreatic cancer development by interfering with the expression of two genes: (1) p53, aiming to induce rapid pancreatic cancer development in combination with mutant Kras, and (2) a gene of interest; in this instance, a candidate therapeutic target.

Using "tandem" shRNA technology developed in our laboratory that links two shRNAs in the same transcript (Chicas et al. 2010), it is possible to inactivate two genes, essentially replacing the requirement for four conditional knockout alleles. To first test whether single-copy expression of two shRNAs in tandem from a single genomic copy could result in potent inhibition of both targets, we generated a targeting cassette expressing a p53 shRNA and an shRNA targeting Rpa3, a control gene that is necessary for replication in various cell types (shRpa3) (Fig. 5A; McJunkin et al. 2011; Zuber et al. 2011). Following RMCE, ESCs were treated with a limiting titer of adenoviral Cre to obtain a mixed population of $\mathrm{rtTA}_{3}$ and non-rtTA $\mathrm{A}_{3}$-expressing ESCs. shRNA expression upon dox addition was assessed by monitoring the percentage of GFP-positive cells over time. As expected, ESCs expressing shp53/shRpa3 were depleted from the population relative to shp53/shRen (a neutral control shRNA) ESCs, demonstrating effective inhibition of Rpa3 from the tandem shRNA expression cassette (Fig. 5A).

To test the approach in an in vivo setting, we generated shp53/shRen and shp53/shRpa3 chimeric mice by 
A
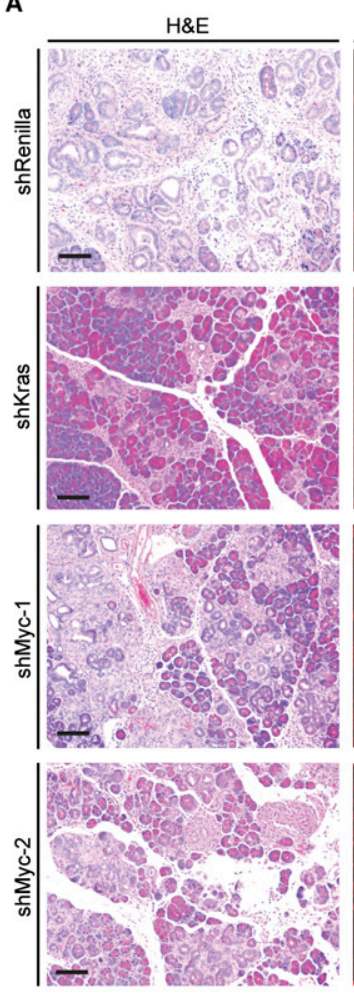

B

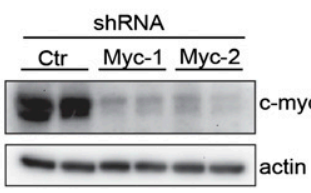

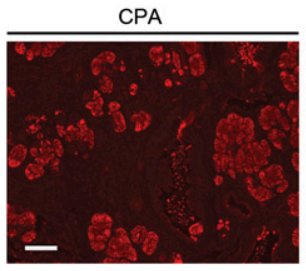
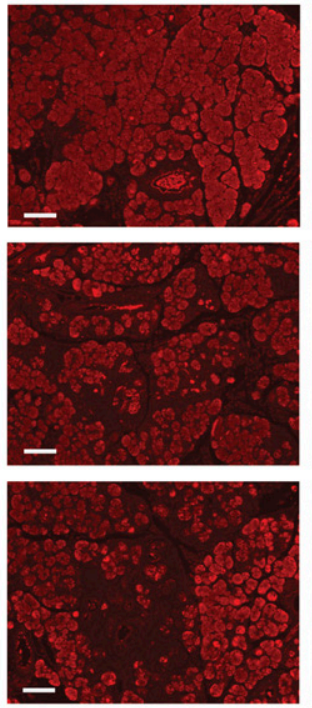
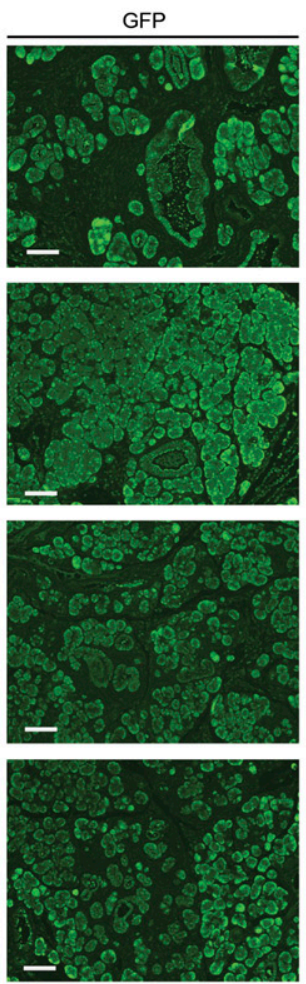
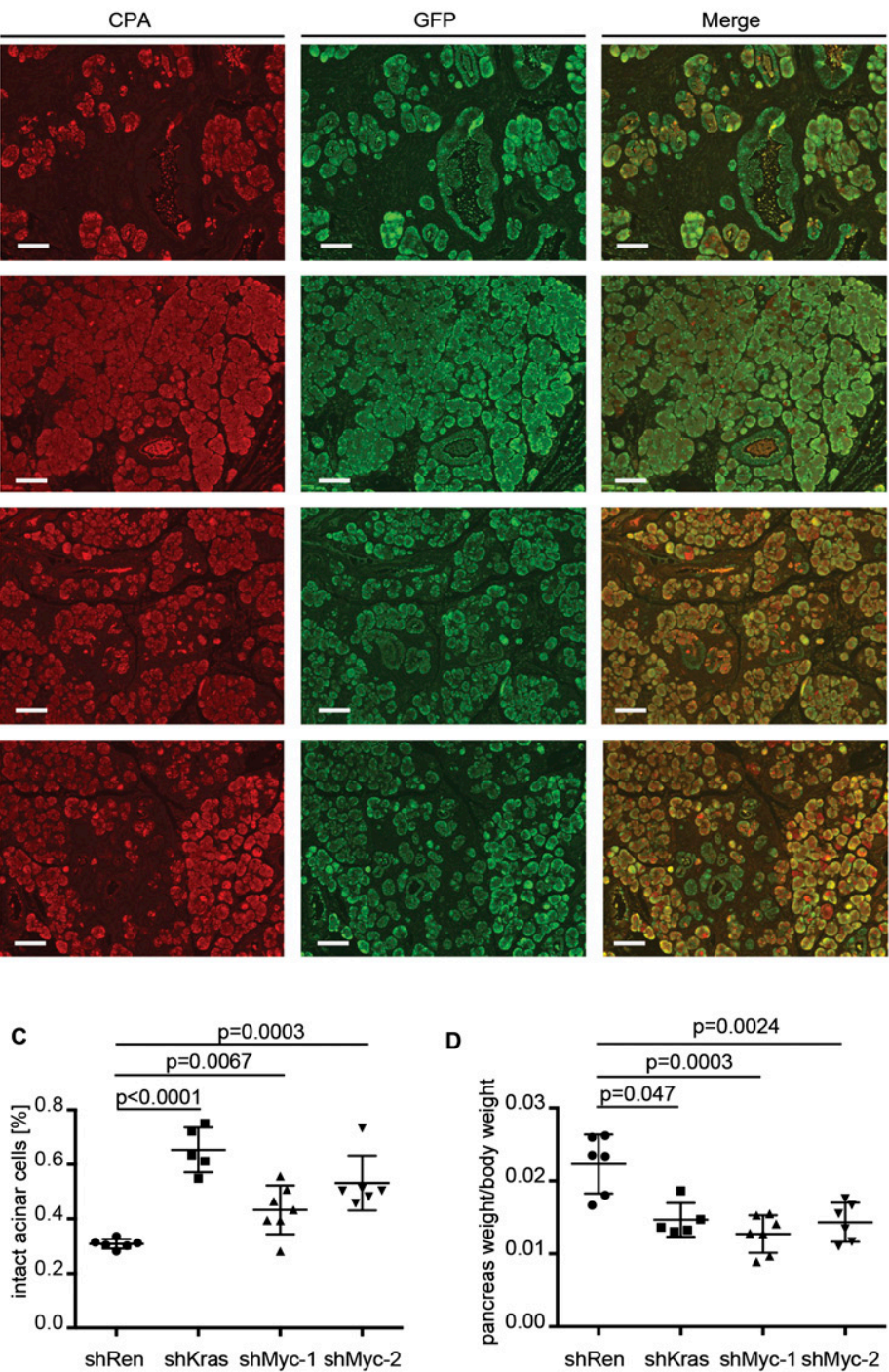

D

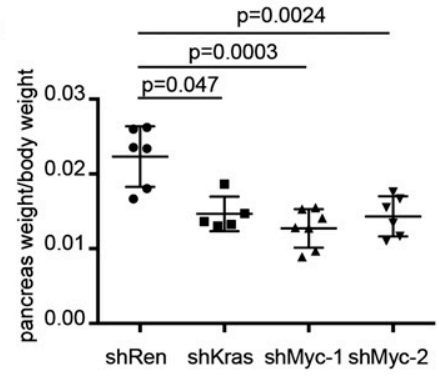

Figure 4. $\mathrm{c}-\mathrm{Myc}$ plays a major role in $\mathrm{Kras}^{\mathrm{G} 12 \mathrm{D}}$-induced neoplasia. $(A) \mathrm{H} \& \mathrm{E}$ and immunofluorescence for GFP and carboxypeptidase $\mathrm{Al}$ (CPA) from pancreata of p48 GEMM-ESC mice expressing shRNAs directed against Renilla, Kras, and c-Myc after 4 wk on dox feed, starting on postnatal day 11. (B) Immunoblot confirming knockdown of c-Myc in sorted GFP- and mKate2-positive adult pancreatic cells from dox-treated mice. $(C)$ Knockdown of Kras and c-Myc leads to retention of intact acinar tissue, as assessed by CPA-positive over total pancreatic area. $(D)$ Decreased pancreas/body weight ratio in shMyc and shKras mice. Bars, $100 \mu \mathrm{m}$.

blastocyst injection. Resulting animals were treated with dox from 1 wk after birth to induce shRNA expression. Mice harboring the shp53/shRen vector succumbed to pancreatic tumors with a median latency of $60 \mathrm{~d}$. In contrast, mice expressing shp53/shRpa3 did not develop disease within 5 mo (Fig. 5B). Histologically, tumors of shp53/shRen mice were predominantly undifferentiated adenocarcinomas with sarcomatoid features and focal glandular differentiation (Fig. 5G,H). GFP- and mKate2positive cells were present in the pancreas in both experimental colonies at 5-6 wk (Fig. 5C,D) as well as in tumors that developed in shp53/shRen mice (Fig. 5E). However, shp53/shRpa3 mice harvested 19 wk after dox treatment contained almost no mKate2/GFP-positive cells within the pancreas, consistent with the elimination of these cells by Rpa3 knockdown (Fig. 5F).
c-Myc inhibition suppressed $\mathrm{Kras}^{G 12 D}$-driven ADM and PanIN (Fig. 4). We set out to test whether c-Myc expression is required for the later stages of pancreatic cancer formation. Importantly, knockdown efficiency in ESCs was comparable between single shRNAs targeting $c-M y c$ (shMyc-1 and shMyc-2) and the tandem construct (shp53/ shMyc) (Fig. 6A). As expected, shp53/shRen chimeric mice generated by blastocyst injection succumbed to pancreatic cancer (median latency of $66 \mathrm{~d}$ ) with full penetrance. In contrast, knockdown of c-Myc in this context significantly improved survival of shp53/shMyc-expressing mice (Fig. 6B). Some of the shp53/shMyc mice eventually developed pancreatic cancer at significantly longer latencies, which strongly expressed mKate 2 and GFP and presented as poorly differentiated or undifferentiated carcinomas, similar to tumors from shp53/shRen mice (Fig. 6B,C). In summary, 
Saborowski et al.

A
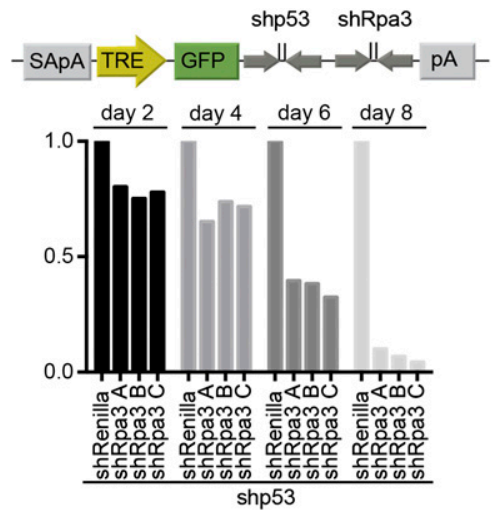

C

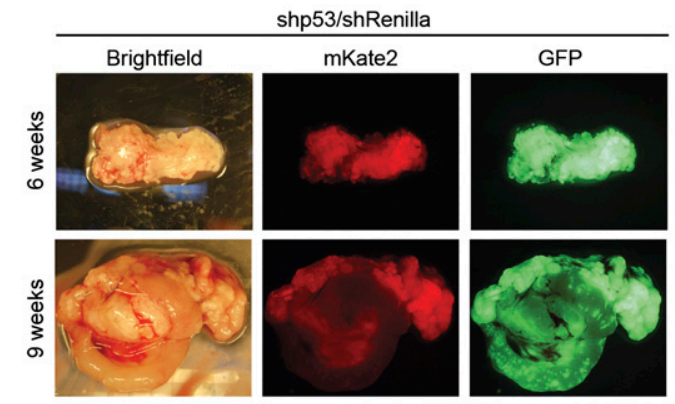

G

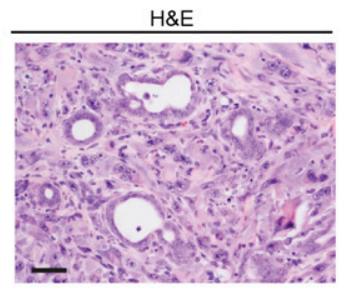

H
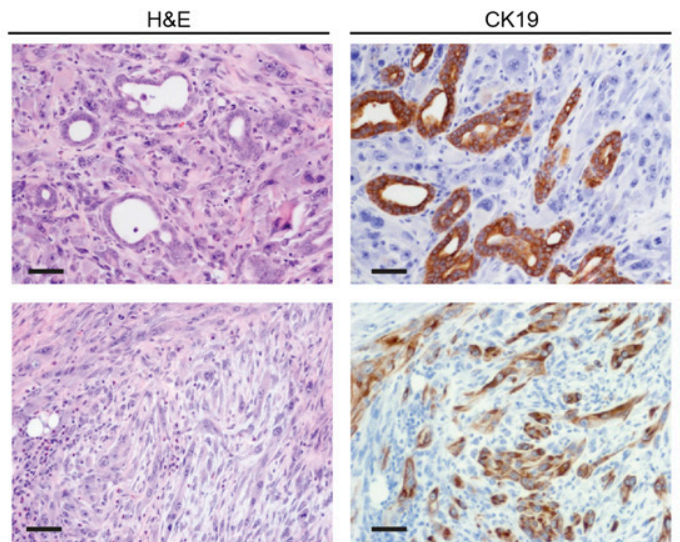

B

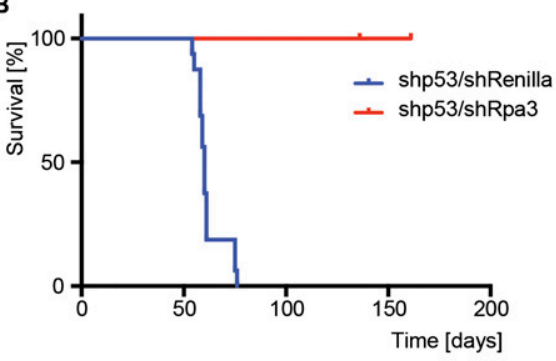

D

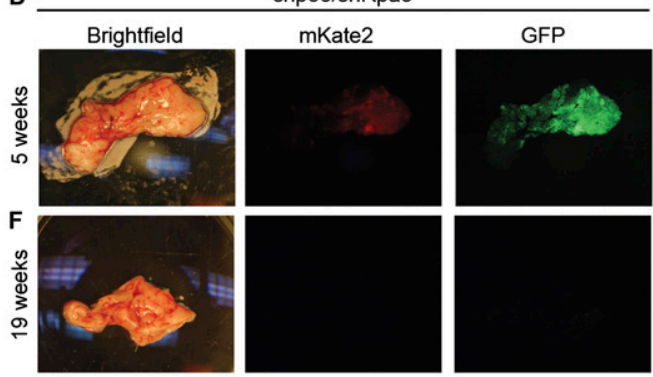

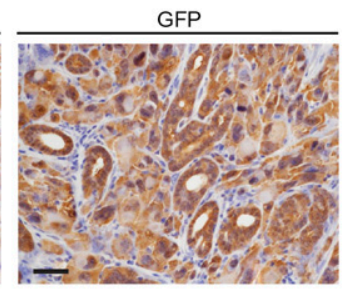
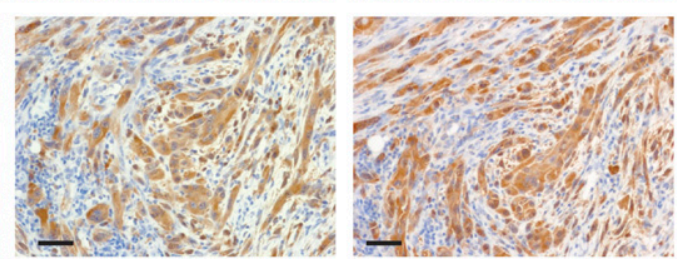

Figure 5. The tandem cTGM targeting vector allows for combined inhibition of two independent genes. $(A)$ Schematic of the tandem cTGM targeting vector. Depletion of shp53/shRpa3-expressing relative to shp53/shRenilla-expressing Pdx1 ESC clones functionally confirmed inhibition of $R p a 3$ by shRpa3 in the second position. Pdx1 ESCs were treated with adenoviral Cre recombinase to activate the latent $\mathrm{rtTA}_{3}$ within the CAGS-LSL-RIK and were subsequently treated with dox-containing growth medium. $(B) \mathrm{KM}$ survival curve of chimeric mice generated with the Pdx1 GEMM-ESCs and expressing shp53/shRenilla $(n=16)$ or shp53/shRpa3 $(n=8)$. Mice received doxcontaining feed starting on postnatal day 5. shp53/shRenilla mice succumbed to pancreatic cancer with a median latency of $60 \mathrm{~d}$, while expression of shRpa3 efficiently inhibited pancreatic tumor development. $(C, D)$ At 5-6 wk of age, pancreata of shp53/shRenilla and shp53/ shRpa3 mice expressed mKate2 and GFP as a surrogate for tandem cTGM expression. (E) A tumor from a 9-wk-old shp53/shRenilla mouse expressed mKate2 and GFP. (F) A pancreas from a 19-wk-old shp53/shRpa3 mouse depicts almost complete depletion of mKate2- and GFPexpressing cells from the pancreas. Histopathology from mostly poorly differentiated pancreatic adenocarcinomas that developed in shp53/shRenilla mice are shown in $E$ and $F$. Histopathology from mostly poorly differentiated pancreatic adenocarcinomas that developed in shp53/shRenilla mice are shown in $G$ and $H$. Pdx1-Cre recombined cells can be identified by mKate2 and GFP expression. Bars, $50 \mu \mathrm{m}$.

using our GEMM-ESCs, we were able to efficiently demonstrate a requirement of c-Myc for Kras ${ }^{\mathrm{G} 12 \mathrm{D}}$ and p53 lossmediated induction of pancreatic cancer.

\section{Discussion}

GEMMs of pancreatic cancer provide a powerful platform to study cancer initiation and maintenance, the tumor microenvironment, and the responsiveness of tumors to known or novel therapeutics. Despite their theoretical value, the time and cost required to model the effect of gene combinations on PDAC progression and maintenance limits their practical utility. We report the generation of two GEMM-ESC lines that facilitate the rapid production of chimeric mice harboring pancreas-specific Cre recombinases (Pdx1-Cre and p48-Cre) in combination 
A

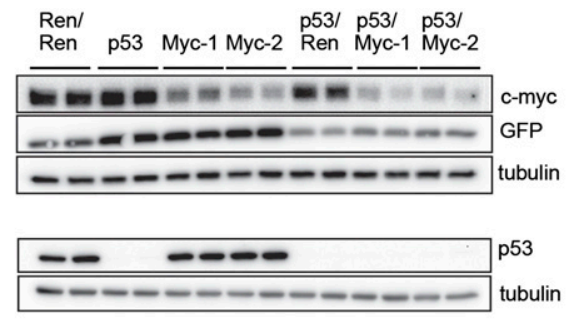

C
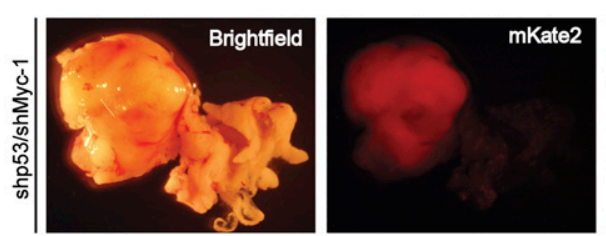
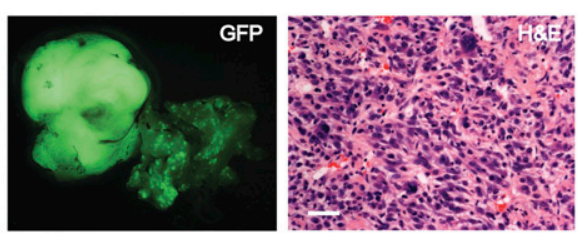

Figure 6. Knockdown of c-Myc impairs pancreatic cancer development. (A) Immunoblot demonstrating that the efficiency of target gene knockdown is equally potent upon introduction of single or tandem shRNAs into the CHC locus. (B) c-Myc depletion by shp53/shMyc-1 and shp53/shMyc-2 leads to a significant survival benefit and strongly impairs pancreatic cancer development $(P=0.0006 ; P<0.0001$, respectively; shp53/shRen, $n=11$; shp53/shMyc-1, $n=5$; shp53/shMyc-2, $n=10$ ). Mice expressing shp53/shMyc-1 and shp53/shMyc-2 do not show a significant difference in survival $(P=0.1988)$. $(C)$ Tumors developing in shp53/shMyc-1-expressing mice are positive for mKate 2 and GFP and are of poorly differentiated histology (comparable with shp53/shRen tumors). Bars, $50 \mu \mathrm{m}$.

with a Cre-activated $\mathrm{Kras}^{G 12 D}$ allele, thus enabling pancreas-specific expression of the hallmark oncogenic lesion of the disease. The additional incorporation of the col1a1 targeted "CHC" locus and CAGs-LSL-RIK allele allows for high-efficiency targeting with shRNAs and dox-dependent gene silencing in Cre-recombined cells, thus enabling conditional modulation of endogenous gene expression at different stages of $\mathrm{Kras}^{\mathrm{G} 12 \mathrm{D}}$-induced disease.

Our models incorporate a dual fluorescent reporter system that provides additional flexibility and power in experimental analyses of the resulting mice. Specifically, the CAGs-LSL-rtTA ${ }_{3}$-IRES-mKate2 (CAGs-LSL-RIK) allele that is used to produce cells capable of inducing tetresponsive transgenes coexpresses a linked mKate 2 reporter, thereby facilitating the precise identification of cells that have undergone Cre-mediated recombination in tissue by far-red fluorescence. Similarly, the GFP reporter linked to the shRNA facilitates the identification of cells in which the shRNA is expressed $\left(\mathrm{mKate}^{+} / \mathrm{GFP}^{+}\right)$ and those in which it has been silenced $\left(\mathrm{mKate}^{+} / \mathrm{GFP}^{-}\right)$. These reporters also provide an additional level of quality control that empowers subsequent analysis; for example, determining whether cancers that ultimately progress arise from cells in which the shRNA was not sufficiently silenced $\left(\mathrm{GFP}^{+}\right)$or was never expressed $\left(\mathrm{GFP}^{-}\right.$, $\left.\mathrm{mKate}^{-}\right)$or from cells that are truly resistant to the effects of gene reactivation $\left(\mathrm{GFP}^{-}\right.$, mKate $\left.2^{+}\right)$.

The system is robust and permits rapid production of experimental cohorts of mice that develop a disease identical to the corresponding germline model without intercrossing strains. Thus, one round of standard blastocyst injection ( $~ 50-60$ transferred blastocysts) produces $\sim 20$ mice_enough animals for most experimental assessments of tumor growth and/or therapy response. Indeed, with these ESCs in hand, production of experimental mice in which any gene can be assessed for its impact at different stages of PDAC progression can be $<2$ mo (Supplemental Fig. S1A), reducing the time required to investigate gene function in a multiallelic genetic background by nearly 10 -fold. Additionally, the RMCE-based gene targeting approach enables the study of several genes in parallel. As the ESCs contribute to pancreatic development, experiments can be conducted in a mosaic setting in which normal cells also contribute to the analyzed tissue. On the one hand, this mimics the human cancer microenvironment, where both cancer cells and normal cells are present and interact; however, on the other hand, the varied chimerism could mask subtle effects of gene perturbations on tumor onset due to increased variability. Still, for some applications, it may be useful to limit the number of injected ESCs, allowing a greater fraction of the animal to be derived from the injected blastocyst.

We used these GEMM-ESC models to study the role of gene suppression at different stages of tumorigenesis and also the consequences of gene reactivation at later stages of disease. To do this, we took advantage of the flexible mir30-based shRNA system that has been optimized by our laboratory and others over the last several years (Dickins et al. 2005; Silva et al. 2005; Zuber et al. 2011). Besides the ability to link these shRNAs to fluorescent reporters, shRNAs can also be physically linked in "tandem," enabling cosuppression of multiple alleles. As shown here, this cosuppression can be robust and, in essence, produces effects that recapitulate a "double knockout" from a single expression construct. Importantly, the GEMM-ESC models proposed here are not limited to shRNAs but can include cDNAs, microRNAs, or indeed any genetic element.

A major limitation of current GEMMs relates to the challenge of genetically validating novel therapeutic 
targets. This presents an urgent need in pancreas cancer, where studies clearly document the importance of the in vivo microenvironment on PDAC development and therapy response. Current methods to perform such validation require the introduction of conditional knockout alleles into the $\mathrm{Kras}^{G 12 D}$ or $\mathrm{Kras}^{\mathrm{G12D}} /$ mutant p53 model (KPC) and assessment of subsequent tumor formation in the presence and absence of gene function. While a powerful approach, execution of such experiments requires the presence of an appropriate conditional knockout animal followed by the generation of whole cohorts of mice harboring at least five alleles. Furthermore, as the putative target is necessarily deleted concurrently with Kras $^{G 12 D}$ activation when Cre is expressed during embryogenesis, its impact on PDAC can be assessed only at the initiation stage and only if it plays no role in normal pancreas development. By uncoupling analysis of the target gene (which is controlled by dox) from oncogene activation (controlled by Cre), the GEMM-ESC model enables the impact of target inhibition to be assessed after pancreas development and the onset of disease.

In one application of the model, we probed the requirement for sustained Pten loss in PDAC initiation and maintenance. As was observed in germline transgenic mice harboring conditional $\mathrm{Kras}^{\mathrm{G1} D}$ and floxed Pten alleles (Hill et al. 2010; Ying et al. 2011), the GEMMESC chimeras displayed accelerated tumor onset. Importantly, tumor-bearing animals showed potent suppression of Pten in pancreatic epithelium, which corresponded to the expression of mKate2 (rtTA (shRNA-linked) reporters. Upon tumor formation, withdrawal of dox triggered silencing of the GFP reporter and restoration of normal PTEN levels, clearly documenting the robustness of the system. Although pancreas histology did not revert back to normal, tumors shrank rapidly and did not reoccur over 3 mo. Interestingly, in a lymphoma model, the impact of PTEN reactivation on disease maintenance was blunted in particular microenviroments, suggesting that both cell-autonomous and cell-nonautonomous mechanisms can contribute to its effects (C Miething and SW Lowe, in prep.). Although further studies are required to explore how re-establishing control over the PI3K pathway promotes tumor regression, our results show promise for the use of PI3K inhibitors in PDAC. They also provide a proof of concept illustrating the power of the GEMM-ESC approach to study cancer drivers in cancer initiation and maintenance.

In another application, we tested the suitability of the system for validating therapeutic targets. This represents a particularly challenging experimental context, as it is desirable that target inhibition should occur after Kras oncogene activation to preclude potential effects on pancreas development; moreover, for those genes whose inhibition produces a strong anti-cancer effect, there is pressure for mutations to disable the elements needed for gene silencing and the rapid emergence of cells lacking the shRNA. As proof of principle, we assessed the consequences of inhibiting Kras or $c-M y c$ at early stages of disease development and the effects of inhibiting Rpa3 and $c-M y c$ on preventing advanced disease. These studies provide decisive evidence that c-Myc is important for PDAC initiation and progression independent of its role in pancreas development.

Several features of the GEMM-ESC platform facilitate the conduct and interpretation of such studies. First, since the production and targeting of shRNA vectors into ESCs is straightforward, it is possible to analyze the impact of multiple candidate targets in parallel. Second, since $p 53$ mutations potently cooperate with Kras $^{\text {G12D }}$ during PDAC development, the use of tandem shRNAs linking an experimental shRNA to a second shRNA targeting $p 53$ provides a strong selective pressure against shRNA silencing. By examining dox-treated tissues for the shRNA-linked GFP reporter, it should be possible to determine whether any lesions that form have silenced the shRNA (i.e., are $\mathrm{GFP}^{-}$). For example, while inactivation of the essential Rpa3 gene in our experiments led to loss of $\mathrm{Kate}^{+} / \mathrm{GFP}^{+}$cells, $\mathrm{Kate}^{+} / \mathrm{GFP}^{+}$tumors eventually arose in shp53/shMyc mice, suggesting evasion from the effects of $c-M y c$ inhibition rather than silencing of the transgenic locus. Producing the equivalent genetic context using a traditional approach would require laborious breeding to incorporate six alleles (i.e., Pdx1-Cre; LSLKras $^{G 12 D} ; c-M y c^{\text {lox/lox }} ; p 53^{\text {lox } / l o x} \mid$ to characterize the role of a single gene and would lack any fluorescent reporters of system functionality. Thus, the GEMM-ESC approach, in combination with shRNA technology, provides the means to investigate the role of multiple candidate genes simultaneously in a time scale of months rather than years.

Beyond its use for genetic studies, these and other GEMM-ESC models will facilitate the production of large animal cohorts harboring distinct genetic lesions for preclinical drug evaluation. Currently, such efforts involve the maintenance of relatively large breeding colonies to produce a steady supply of animals with an appropriate genotype; unless breeding is continuous, there is a long wait time to obtain sufficient mice, and the study must be conducted in a staggered fashion as mice can be enrolled. With the GEMM-ESC approach, mouse cohorts of the appropriate genotype and colony size can be produced simultaneously to coincide with a specific study. Additionally, the far-red mKate2 fluorescent reporter complements the use of ultrasound for monitoring tumor responses to therapy and should enable intravital microscopy of tumor responses as well as specific sorting of PDAC cells to assess aspects of treatment response. Given the complexity of the PDAC microenvironment, such models should greatly accelerate drug development.

The high portability of GEMM-ESCs should increase the accessibility of these models in the research community. ESC-based models can easily be transferred between institutes, usually avoiding quarantine. Moreover, they offer a means for almost any laboratory to examine their gene of interest in a complex yet well-defined in vivo setting without needing to house and maintain a large animal colony. The models described here provide a blueprint for models of other cancer types or indeed other types of disease, and new genome-editing technologies 
will expedite their production. Indeed, we are already producing GEMM-ESC models of PDAC with Kras and p53 mutations or Ink4a/Arf deletions that should be particularly valuable for target validation in advanced cancers as well as GEMM-ESCs to model cancers in several other tissue types. By changing the diseasepromoting alleles engineered in the ESCs and the tissue-specific Cre, it is possible to study gene perturbations in virtually any cancer model or human disease. We believe that such approaches will accelerate our understanding of genetic interactions in cancer and facilitate the identification and validation of novel therapeutic targets.

\section{Materials and methods}

\section{Generation of ESC lines}

ESC lines were generated as described previously (Bryja et al. 2006) with the following modifications. Transgenic female mice 3-6 wk old were superovulated and mated with multiallelic male mice. Blastocysts were isolated at embryonic day 3.5 and incubated in a $80-\mu \mathrm{L}$ drop of KSOM+AA (Millipore) under mineral oil for 7-8 hr. After a brief wash in M2 medium (Millipore), blastocysts/ESC clones were cultured and expanded in KOSR $+2 \mathrm{i}$ medium (Gertsenstein et al. 2010) on irradiated mouse embryonic fibroblast (MEF) feeder layers until they reached $70 \%$ confluency on a well of a six-well plate. For passaging of ESC clones, we used ESGRO Complete Accutase (Millipore). ESC clones were genotyped and cryopreserved. Male ESC clones with the correct genotype were further expanded in M15+LIF medium (for detailed recipe, see Dow et al. 2012).

\section{Targeting the CHC locus}

ESC lines were thawed into M15+LIF medium and cultured for 2 $\mathrm{d}$ prior to targeting. CHC targeting was performed as described previously (Dow et al. 2012). The Pdx1 ESC line was cultured in KOSR $+2 \mathrm{i}$ medium (Gertsenstein et al. 2010) starting immediately after targeting and also throughout selection with hygromycin (Roche). The p48 ESC line was kept in M15+LIF and changed to KOSR $+2 \mathrm{i}$ no earlier than $48 \mathrm{~h}$ prior to blastocyst injection.

Correct integration of the targeting vector into the $\mathrm{CHC}$ locus was confirmed by PCR as described previously (Dow et al. 2012). To rule out additional random integrations of the targeting vector in the genome, we performed a TaqMan copy number assay for GFP (Invitrogen) according to the manufacturer's instructions on a ViiA7 RT-PCR machine (Life Technologies). Functionally, ESC clones were tested by treatment with and without adenoviral Cre recombinase (University of Iowa) to activate the latent $\mathrm{rtTA}_{3}$ within the CAGs-LSL-RIK allele in the adenoviral Cre-treated ESCs and by subsequent addition of dox to the growth medium for $3 \mathrm{~d}$, and GFP induction was detected by flow cytometry to determine activation of the targeted CHC locus.

\section{Mouse strains}

All animal experiments were performed in accordance with a protocol approved by the Memorial Sloan-Kettering Institutional Animal Care and Use Committee.

All mouse strains have been previously described. Pdx1-Cre (Hingorani et al. 2003), p48-Cre (Kawaguchi et al. 2002), LSLKras $^{G 12 D}$ (Jackson et al. 2001), LSL-p53 ${ }^{\text {R172H }}$ (Olive et al. 2004), CHC (Beard et al. 2006), and CAGs-LSL-RIK strains (LE Dow, Z Nasr, M Saborowski, SH Ebbesen, E Manchado, N Tasdemir,
J Pelletier, and SW Lowe, in prep.) were interbred and maintained on mixed background. Dox feed $(625 \mathrm{mg} / \mathrm{kg})$ purchased from Harlan Laboratories was replenished twice weekly. Chimeric mice were generated by the Mouse Genetics Core-Transgenic Core of Memorial Sloan-Kettering Cancer Center (MSKCC). For unknown reasons, a subset of mice from both GEMM-ESC lines developed nonpancreatic illnesses; $15 \%-20 \%$ of the Pdx1 GEMMESC mice developed hydrocephalus at $\sim 3-6$ wk of age, and $<10 \%$ of the p48 GEMM-ESC-containing mice developed neurological phenotypes characterized by a tilted head. These mice were euthanized and excluded from the analysis. For experiments with shRNA-expressing p48 GEMM-ESC mice, we included mice with a coat color chimerism $>90 \%$. For tandem vector-expressing Pdx 1 GEMM-ESC mice, we used mice with $>30 \%$ coat color chimerism. Tumor volume was followed by three-dimensional ultrasound imaging (Visualsonics, Vevo 2000).

\section{Array CGH}

Genomic DNA from p48 and Pdx1 GEMM-ESCs was compared with C57BL/6J reference genomic DNA by the MSKCC Genomic Core Laboratory (GCL) using SurePrint G3 1M Mouse CGH microarray kits (Agilent). As ESCs were derived from mice on a partially mixed background containing 129 segments $\mid<20 \%$ by 384 SNP array), an additional $1 \mathrm{M}$ array CGH comparison of C57BL/6J with 129S4/SvJae genomic DNA was included in the analysis. Image data were extracted with Agilent Feature Extraction software, and data were normalized using the MSKCC Bioinformatics Core Facility algorithm for array CGH (http:// cbio.mskcc.org/CGCC) by the GCL. Amplifications and deletions (CNVs) were called using the ADM-2 algorithm with threshold 6.0, GC correction on, window size $2 \mathrm{~kb}$, minimum number of probes three or more, minimum size (in kilobases) of region $\geq 0.0$, and minimum average absolute $\log _{2}$ ratio $\geq 0.5$ using Agilent Genomic Workbench 7.0. Of the total 285 CNVs called in p48 and Pdx GEMM-ESCs, CNVs in non-gene-coding regions (27 CNVs; $9.5 \%$ of total CNVs) or co-occurring in 129S4/ SvJae genomic DNA and/or the other ESC line (228 CNVs; $80 \%$ of total CNVs) were excluded from Supplemental Table 1.

\section{Immunohistochemistry, immunoblotting, and immunofluorescence}

For immunohistochemistry, slides were heated for 30-50 min at $55^{\circ} \mathrm{C}$, deparaffinized, and rehydrated via stepwise alcohol washes. Antigen retrieval was performed in citrate buffer, and primary antibodies were incubated overnight at $4^{\circ} \mathrm{C}$. Anti-cytokeratin 19 (Epitomics, no. 3863-1), anti-GFP (Cell Signaling Technology, no. 2956), anti-Pten (Cell Signaling Technology, no. 9188S), and antitRFP (Evrogen, no. AB233-234). For immunofluorescence, the following primary antibodies were used: anti-GFP (Abcam, no. ab13970), anti-carboxypeptidase A1 (R\&D Systems, no. AF2765), and anti-tRFP (Evrogen, no. AB233-234). Secondary antibodies used were as follows: Alexa Fluor 594 donkey anti-goat (Invitrogen, no. A11058), Alexa Fluor 488 donkey anti-chicken IgY (Jackson ImmunoResearch, no. 703-546-155), DyLight 488 goat anti-chicken (Abcam, no. 96947), and Alexa Fluor 568 goat anti-rabbit (Invitrogen, no. A11036).

For immunoblotting, cells were washed in cold phosphatebuffered saline (PBS) and lysed on ice in cell lysis buffer (Cell Signaling Technology, no. 9803). All primary antibodies were incubated overnight at $4{ }^{\circ} \mathrm{C}$ in Tris-buffered saline $/ 5 \%$ milk/ $0.1 \%$ Tween20. HRP-anti- $\beta$-actin (Sigma, no. A3854), anti-GFP (Cell Signaling Technology, no. 2956), anti-c-Myc (Cell Signaling Technology, no. D84C12), (Epitomics, no. 1472-1), anti-p53 (Leica Biosystems, no. NCL-p53-505), and anti-tubulin (Sigma, no. t5168) were also used. 


\section{Isolating single cells from mouse pancreata}

Single pancreatic cells were isolated from 2-4 wk old mice with a modified version of the protocol used by Sugiyama et al. (2007). Pancreata were minced and sequentially incubated with $2 \mathrm{mg} /$ $\mathrm{mL}$ collagenase D (in Hanks buffered saline solution; Roche), $0.05 \%$ trypsin (Invitrogen), and $2 \mathrm{U} / \mathrm{mL}$ dispase (Invitrogen). Dnase 1 (100 ug/mL; Sigma) was included in buffers used for enzyme incubations. Preparations were washed with PBS after the collagenase incubation and with FACs buffer (2\% FBS, 10 mM EGTA, in PBS) after trypsin and dispase digestion. Suspensions were then filtered through $40 \mu \mathrm{M}$ mesh and resuspended in FACs buffer containing $300 \mathrm{nM}$ DAPI as a live-cell marker. Sorts were performed on a FacsARIAIII (Becton Dickinson) for GFP and mKate2.

\section{Statistical analysis}

Statistical analysis was performed using GraphPad Prism software by applying the Log-rank (Mantel-Cox) test to compare survival data and the unpaired two-tailed $t$-test for all other experimental data.

\section{Acknowledgments}

We thank Danielle Grace and Janelle Simon for expert technical assistance. This work was supported by fellowships from the Mildred Scheel Stiftung (Antragsnummer 108734) (M.S.), the German Research Foundation (Post-doctoral Scholarship no. 2278/1-1) (A.S.), a program project grant from the National Cancer Institute (P01-CA013106), the Cancer Target Discovery and Development (CTDD) consortium (NCI), and the Lustgarten Foundation. S.W.L. is an investigator in the Howard Hughes Medical Institute and the Geoffrey Beene Chair of Cancer Biology.

\section{References}

Aguirre AJ, Bardeesy N, Sinha M, Lopez L, Tuveson DA, Horner J, Redston MS, DePinho RA. 2003. Activated Kras and Ink4a/ Arf deficiency cooperate to produce metastatic pancreatic ductal adenocarcinoma. Genes Dev 17: 3112-3126.

Ardito CM, Gruner BM, Takeuchi KK, Lubeseder-Martellato C, Teichmann N, Mazur PK, Delgiorno KE, Carpenter ES, Halbrook CJ, Hall JC, et al. 2012. EGF receptor is required for KRAS-induced pancreatic tumorigenesis. Cancer Cell 22: 304-317.

Barcellos-Hoff MH, Lyden D, Wang TC. 2013. The evolution of the cancer niche during multistage carcinogenesis. Nat Rev Cancer 13: 511-518.

Bardeesy N, Aguirre AJ, Chu GC, Cheng KH, Lopez LV, Hezel AF, Feng B, Brennan C, Weissleder R, Mahmood U, et al. 2006a. Both p16(Ink4a) and the p19(Arf)-p53 pathway constrain progression of pancreatic adenocarcinoma in the mouse. Proc Nat1 Acad Sci 103: 5947-5952.

Bardeesy N, Cheng KH, Berger JH, Chu GC, Pahler J, Olson P, Hezel AF, Horner J, Lauwers GY, Hanahan D, et al. 2006b. Smad4 is dispensable for normal pancreas development yet critical in progression and tumor biology of pancreas cancer. Genes Dev 20: 3130-3146.

Beard C, Hochedlinger K, Plath K, Wutz A, Jaenisch R. 2006. Efficient method to generate single-copy transgenic mice by site-specific integration in embryonic stem cells. Genesis 44: 23-28.

Bonal C, Thorel F, Ait-Lounis A, Reith W, Trumpp A, Herrera PL. 2009. Pancreatic inactivation of c-Myc decreases acinar mass and transdifferentiates acinar cells into adipocytes in mice. Gastroenterology 136: 309-319.e9.
Bryja V, Bonilla S, Arenas E. 2006. Derivation of mouse embryonic stem cells. Nat Protoc 1: 2082-2087.

Cantley LC. 2002. The phosphoinositide 3-kinase pathway. Science 296: 1655-1657.

Chicas A, Wang X, Zhang C, McCurrach M, Zhao Z, Mert O, Dickins RA, Narita M, Zhang M, Lowe SW. 2010. Dissecting the unique role of the retinoblastoma tumor suppressor during cellular senescence. Cancer Cell 17: 376-387.

Dickins RA, Hemann MT, Zilfou JT, Simpson DR, Ibarra I, Hannon GJ, Lowe SW. 2005. Probing tumor phenotypes using stable and regulated synthetic microRNA precursors. Nat Genet 37: 1289-1295.

Dickins RA, McJunkin K, Hernando E, Premsrirut PK, Krizhanovsky V, Burgess DJ, Kim SY, Cordon-Cardo C, Zender L, Hannon GJ, et al. 2007. Tissue-specific and reversible RNA interference in transgenic mice. Nat Genet 39: 914-921.

Dow LE, Lowe SW. 2012. Life in the fast lane: Mammalian disease models in the genomics era. Cell 148: 1099-1109.

Dow LE, Premsrirut PK, Zuber J, Fellmann C, McJunkin K, Miething C, Park Y, Dickins RA, Hannon GJ, Lowe SW. 2012. A pipeline for the generation of shRNA transgenic mice. Nat Protoc 7: 374-393.

Eser S, Reiff N, Messer M, Seidler B, Gottschalk K, Dobler M, Hieber M, Arbeiter A, Klein S, Kong B, et al. 2013. Selective requirement of $\mathrm{PI} 3 \mathrm{~K} / \mathrm{PDK} 1$ signaling for Kras oncogenedriven pancreatic cell plasticity and cancer. Cancer Cell 23: 406-420.

Fellmann C, Zuber J, McJunkin $\mathrm{K}$, Chang $\mathrm{K}$, Malone CD, Dickins RA, Xu Q, Hengartner MO, Elledge SJ, Hannon GJ, et al. 2011. Functional identification of optimized RNAi triggers using a massively parallel sensor assay. Mol Cell 41: 733-746.

Frese KK, Tuveson DA. 2007. Maximizing mouse cancer models. Nat Rev Cancer 7: 645-658.

Gertsenstein M, Nutter LM, Reid T, Pereira M, Stanford WL, Rossant J, Nagy A. 2010. Efficient generation of germ line transmitting chimeras from C57BL/6N ES cells by aggregation with outbred host embryos. PLOS ONE 5: e11260.

Gittes GK. 2009. Developmental biology of the pancreas: A comprehensive review. Dev Biol 326: 4-35.

Hanahan D, Weinberg RA. 2011. Hallmarks of cancer: The next generation. Cell 144: 646-674.

Heiser PW, Cano DA, Landsman L, Kim GE, Kench JG, Klimstra DS, Taketo MM, Biankin AV, Hebrok M. 2008. Stabilization of $\beta$-catenin induces pancreas tumor formation. Gastroenterology 135: 1288-1300.

Hemann MT, Fridman JS, Zilfou JT, Hernando E, Paddison PJ, Cordon-Cardo C, Hannon GJ, Lowe SW. 2003. An epi-allelic series of p53 hypomorphs created by stable RNAi produces distinct tumor phenotypes in vivo. Nat Genet 33: 396-400.

Heyer J, Kwong LN, Lowe SW, Chin L. 2010. Non-germline genetically engineered mouse models for translational cancer research. Nat Rev Cancer 10: 470-480.

Hidalgo M. 2010. Pancreatic cancer. N Engl J Med 362: 16051617.

Hill R, Calvopina JH, Kim C, Wang Y, Dawson DW, Donahue TR, Dry S, Wu H. 2010. PTEN loss accelerates KrasG12Dinduced pancreatic cancer development. Cancer Res 70: 7114-7124.

Hingorani SR, Petricoin EF, Maitra A, Rajapakse V, King C, Jacobetz MA, Ross S, Conrads TP, Veenstra TD, Hitt BA, et al. 2003. Preinvasive and invasive ductal pancreatic cancer and its early detection in the mouse. Cancer Cell 4: 437-450.

Hingorani SR, Wang L, Multani AS, Combs C, Deramaudt TB, Hruban RH, Rustgi AK, Chang S, Tuveson DA. 2005. Trp53R172H and KrasG12D cooperate to promote chromosomal 
instability and widely metastatic pancreatic ductal adenocarcinoma in mice. Cancer Cell 7: 469-483.

Huijbers IJ, Krimpenfort P, Berns A, Jonkers J. 2011. Rapid validation of cancer genes in chimeras derived from established genetically engineered mouse models. Bioessays 33: 701-710.

Jackson EL, Willis N, Mercer K, Bronson RT, Crowley D, Montoya R, Jacks T, Tuveson DA. 2001. Analysis of lung tumor initiation and progression using conditional expression of oncogenic K-ras. Genes Dev 15: 3243-3248.

Jacobetz MA, Chan DS, Neesse A, Bapiro TE, Cook N, Frese KK, Feig C, Nakagawa T, Caldwell ME, Zecchini HI, et al. 2013. Hyaluronan impairs vascular function and drug delivery in a mouse model of pancreatic cancer. Gut 62: 112-120.

Jaiswal BS, Janakiraman V, Kljavin NM, Chaudhuri S, Stern HM, Wang W, Kan Z, Dbouk HA, Peters BA, Waring P, et al. 2009. Somatic mutations in $\mathrm{p} 85 \alpha$ promote tumorigenesis through class IA PI3K activation. Cancer Cell 16: 463-474.

Kawaguchi Y, Cooper B, Gannon M, Ray M, MacDonald RJ, Wright CV. 2002. The role of the transcriptional regulator Ptfla in converting intestinal to pancreatic progenitors. Nat Genet 32: 128-134.

Mazur PK, Einwachter H, Lee M, Sipos B, Nakhai H, Rad R, Zimber-Strobl U, Strobl LJ, Radtke F, Kloppel G, et al. 2010. Notch2 is required for progression of pancreatic intraepithelial neoplasia and development of pancreatic ductal adenocarcinoma. Proc Natl Acad Sci 107: 13438-13443.

McJunkin K, Mazurek A, Premsrirut PK, Zuber J, Dow LE, Simon J, Stillman B, Lowe SW. 2011. Reversible suppression of an essential gene in adult mice using transgenic RNA interference. Proc Natl Acad Sci 108: 7113-7118.

Nakhai H, Siveke JT, Mendoza-Torres L, Schmid RM. 2008. Conditional inactivation of Myc impairs development of the exocrine pancreas. Development 135: 3191-3196.

Olive KP, Tuveson DA, Ruhe ZC, Yin B, Willis NA, Bronson RT, Crowley D, Jacks T. 2004. Mutant p53 gain of function in two mouse models of Li-Fraumeni syndrome. Cell 119: 847-860.

Olive KP, Jacobetz MA, Davidson CJ, Gopinathan A, McIntyre D, Honess D, Madhu B, Goldgraben MA, Caldwell ME, Allard D, et al. 2009. Inhibition of Hedgehog signaling enhances delivery of chemotherapy in a mouse model of pancreatic cancer. Science 324: 1457-1461.

Premsrirut PK, Dow LE, Kim SY, Camiolo M, Malone CD, Miething C, Scuoppo C, Zuber J, Dickins RA, Kogan SC, et al. 2011. A rapid and scalable system for studying gene function in mice using conditional RNA interference. Cell 145: $145-158$.

Provenzano PP, Cuevas C, Chang AE, Goel VK, Von Hoff DD, Hingorani SR. 2012. Enzymatic targeting of the stroma ablates physical barriers to treatment of pancreatic ductal adenocarcinoma. Cancer Cell 21: 418-429.

Schonleben F, Qiu W, Ciau NT, Ho DJ, Li X, Allendorf JD, Remotti HE, Su GH. 2006. PIK3CA mutations in intraductal papillary mucinous neoplasm/carcinoma of the pancreas. Clin Cancer Res 12: 3851-3855.

Silva JM, Li MZ, Chang K, Ge W, Golding MC, Rickles RJ, Siolas D, Hu G, Paddison PI, Schlabach MR, et al. 2005. Secondgeneration shRNA libraries covering the mouse and human genomes. Nat Genet 37: 1281-1288.

Sugiyama T, Rodriguez RT, McLean GW, Kim SK. 2007. Conserved markers of fetal pancreatic epithelium permit prospective isolation of islet progenitor cells by FACS. Proc Natl Acad Sci 104: 175-180.

Vogelstein B, Papadopoulos N, Velculescu VE, Zhou S, Diaz LA Jr, Kinzler KW. 2013. Cancer genome landscapes. Science 339: 1546-1558.
Ying $\mathrm{H}$, Elpek KG, Vinjamoori A, Zimmerman SM, Chu GC, Yan $\mathrm{H}$, Fletcher-Sananikone E, Zhang $\mathrm{H}$, Liu $\mathrm{Y}$, Wang $\mathrm{W}$, et al. 2011. PTEN is a major tumor suppressor in pancreatic ductal adenocarcinoma and regulates an NF-кB-cytokine network. Cancer Discov 1: 158-169.

Zhou Y, Rideout WM 3rd, Zi T, Bressel A, Reddypalli S, Rancourt R, Woo JK, Horner JW, Chin L, Chiu MI, et al. 2010. Chimeric mouse tumor models reveal differences in pathway activation between ERBB family- and KRAS-dependent lung adenocarcinomas. Nat Biotechnol 28: 71-78.

Zuber J, McJunkin K, Fellmann C, Dow LE, Taylor MJ, Hannon GJ, Lowe SW. 2011. Toolkit for evaluating genes required for proliferation and survival using tetracycline-regulated RNAi. Nat Biotechnol 29: 79-83. 


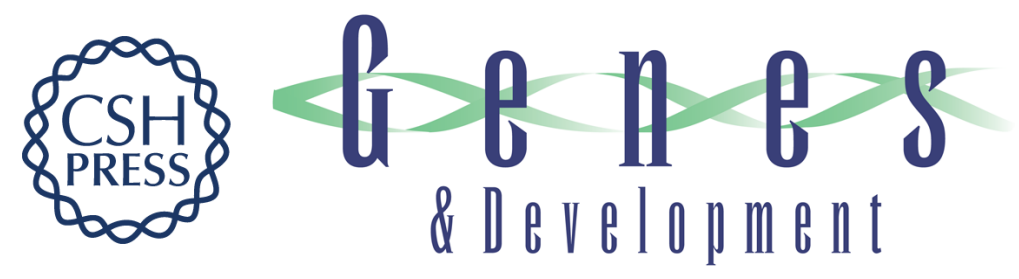

\section{A modular and flexible ESC-based mouse model of pancreatic cancer}

Michael Saborowski, Anna Saborowski, John P. Morris IV, et al.

Genes Dev. 2014, 28:

Access the most recent version at doi:10.1101/gad.232082.113

Supplemental Material

References

Creative Commons License

Email Alerting Service
http://genesdev.cshlp.org/content/suppl/2014/01/06/28.1.85.DC1

This article cites 46 articles, 15 of which can be accessed free at: http://genesdev.cshlp.org/content/28/1/85.full.html\#ref-list-1

This article, published in Genes \& Development, is available under a Creative Commons License (Attribution-NonCommercial 3.0 Unported), as described at http://creativecommons.org/licenses/by-nc/3.0/.

Receive free email alerts when new articles cite this article - sign up in the box at the top right corner of the article or click here.

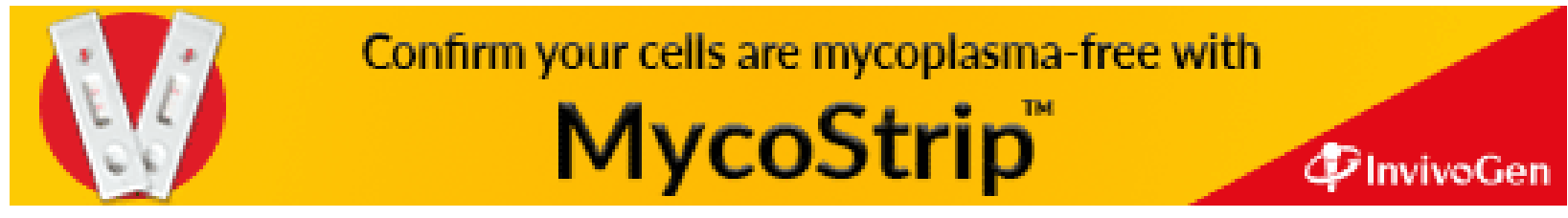

